\title{
Preparation of a PVDF/PVDF-g-(PSSS-co-PAA) graft copolymerization-blending cation exchange material and effects of the synthesis method on the material structures and properties
}

\author{
S. W. Sun ${ }^{*}$, X. L. Gao \\ Key Laboratory of Marine Chemistry Theory and Technology, Ministry of Education, Ocean University of China, 266100 \\ Qingdao, China
}

Received 23 February 2020; accepted in revised form 18 June 2020

\begin{abstract}
In this study, a PVDF/PVDF-g-(PSSS-co-PAA) graft copolymerization-blending cation exchange material was synthesized with polyvinylidene fluoride (PVDF), sodium p-styrene sulfonate (SSS) and acrylic acid (AA) by the methods of solution polymerization and suspension polymerization, based on the preparation of cation exchange materials. Experimental results indicated that the exchange capacity was well designed, and that water absorption and water swelling could be effectively controlled. The morphology of the modified material was analyzed by SEM, which showed that the material had a uniform particle size. Thermal analysis of the material demonstrated that the material synthesized in $N, N$-dimethylformamide (DMF) had excellent thermostability and good compatibility with PVDF.
\end{abstract}

Keywords: polymer composites, graft copolymerization-blending material, suspension polymerization

\section{Introduction}

The price of cation exchange membranes (CEMs) has always been high due to the complex preparation process and expensive ingredients. Thus, a new cation exchange material with a simple film forming process, and inexpensive raw materials is needed. The objective of this paper is to determine the optimum synthesis method for cation exchange materials with application value [11-13]. Polyvinylidene fluoride (PVDF) served as the substrate. Developments in the preparation of cation exchange materials based on PVDF have been reported over the years. PVDF can be modified in mixtures of inorganic $[1,2,8]$ and organic bases [3-5] to generate active sites for grafting reactions. Organic acids and sulfonates have been applied to prepare CEMs as functional materials for a long time $[9,10]$, and these materials include polyacrylic acid (PAA) [6] and sodium p-styrene sulfonate (SSS) [6, 7], which have strong hydrophilicity, electronegativity, and chemical stability. On the other hand, water swelling resulting from hygroscopicity can cause great damage to the forming and application of materials. Therefore, a blending structure that could effectively inhibit water swelling was adopted. In terms of the preparation process, modified cation exchange materials have been prepared by suspension polymerization and solution polymerization methods. The effects of the material formulation and molding methods on the material properties are investigated herein. The material preparation process took into account the hydrophilicity, electrical conductivity, mechanical properties, and water-swelling properties of the material in the membrane formation process, increasing

*Corresponding author, e-mail: eeerrr5345@gmail.com (C) BME-PT 
the application value of the material. This process can solve many problems of cation exchange materials in practical applications, such as the convoluted trade-off between exchange capacity and mechanical properties and insufficient material life.

\section{Experimental}

\subsection{Materials}

Polyvinylidene fluoride (PVDF, FR904) was purchased from Shanghai 3F New Materials Co. (China). $\mathrm{N}, \mathrm{N}$-Dimethylformamide (DMF), acrylic acid, sodium hydroxide, and ammonium persulfate were obtained from Sinopharm Chemical Reagent Co., Ltd. (China). Sodium p-styrene sulfonate (SSS) was supplied by Wengjiang Reagent Co. (China). Divinylbenzene (DVB) was obtained from Aladdin Reagent Co. (China) and distilled under reduced pressure. Dibenzoyl peroxide (BPO) and polyvinylpyrrolidone (PVP, K30) were acquired from Shanghai Macklin Biochemical Co., Ltd. (China). Deionized water was prepared in the laboratory.

\subsection{Preparation}

Ten grams of PVDF was added to $100 \mathrm{ml}$ of $8 \mathrm{~mol} / 1$ aqueous $\mathrm{NaOH}$ solution and heated in a water bath at $80^{\circ} \mathrm{C}$ with high-speed stirring for $8 \mathrm{~h}$. Fifty grams of SSS, $20 \mathrm{~g}$ of AA, $1 \mathrm{~g}$ of PVP, $0.5 \mathrm{~g}$ of ammonium persulfate and $0.2 \mathrm{~g}$ of BPO were dissolved in either $1 \mathrm{~kg}$ of DMF or $1 \mathrm{~kg}$ of deionized water for $2 \mathrm{~h}$ to obtain a homogenous solution. Fifty grams of dried, alkali-treated PVDF was added to this mixture, and the dispersion system was heated in a water bath at $80^{\circ} \mathrm{C}$ for $8 \mathrm{~h}$. Ultimately, the modified material was obtained via filtration and backwashing. P1, which was acquired by drying the deionized water dispersion system, was dissolved in DMF to obtain the M1 casting solution (10 wt\%). CEM M1 was then obtained by a phase-inversion process. The M 2 casting solution was obtained from the reaction conducted in DMF. Subsequently, a phase-inversion process was used to obtain CEM M2. P2 was acquired by drying the M2 casting solution.

\section{Results and discussion \\ 3.1. FTIR of the \\ PVDF/PVDF-g-(PSSS-co-PAA) cation exchange material}

The synthesis of PVDF/PVDF- $g$-(PSSS-co-PAA) was confirmed by Fourier transform infrared (FTIR) spectroscopy. The absorption peaks at 1724.3 and $1726.5 \mathrm{~cm}^{-1}$ in the spectra of $\mathrm{P} 1$ and $\mathrm{P} 2$ are characteristic of $\mathrm{C}=\mathrm{O}$ asymmetric stretching vibrations, and broad peaks for the water molecules incorporated in the hydrophilic material were observed at
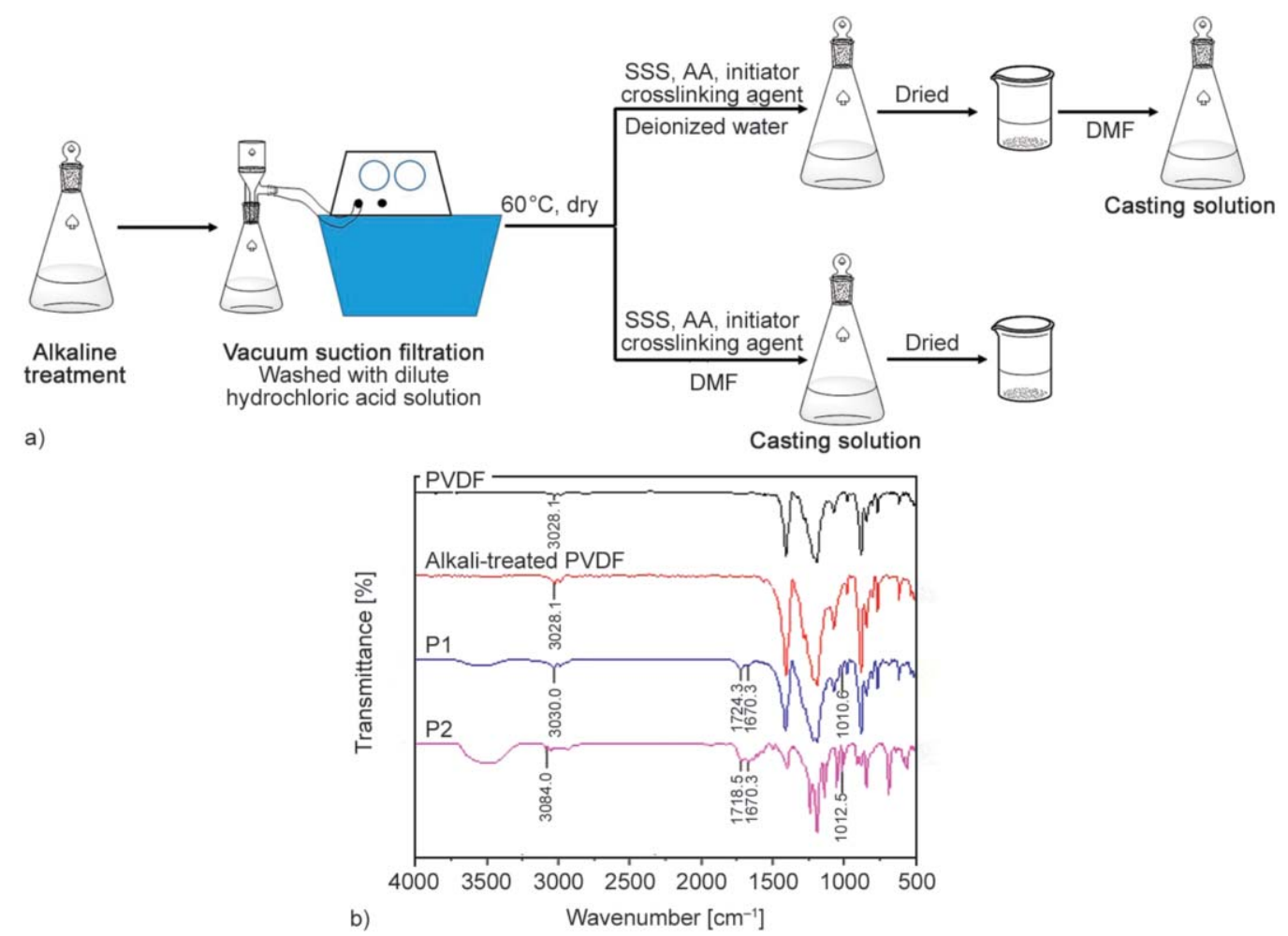

Figure 1. Synthesis and preparation of the PVDF/PVDF-g-(PSSS-co-PAA) graft copolymerization-blending cation exchange material (a) and FTIR spectra of PVDF, alkali-treated PVDF, P1 and P2 (b). 
approximately $3500 \mathrm{~cm}^{-1}$. The stretching vibrations of aromatic functionalities appeared from 1500 $1700 \mathrm{~cm}^{-1}$, and the stretching vibration of $\mathrm{S}=\mathrm{O}$ appeared at 1010 and $1012 \mathrm{~cm}^{-1}$ in the curves of $\mathrm{P} 1$ and $\mathrm{P} 2$, confirming that during polymerization, AA and SSS were successfully grafted onto the alkalitreated PVDF. The absorption band at $1400 \mathrm{~cm}^{-1}$ was attributed to bending vibrations of $\mathrm{C}-\mathrm{H}$, and the pair of absorption bands at 1190 and $1215 \mathrm{~cm}^{-1}$ was caused by stretching vibrations of $\mathrm{C}-\mathrm{F}$, which indicated that the PVDF was not fully reacted in the material modification process. The resulting product was the modified material combined with PVDF.

\subsection{STA of the \\ PVDF/PVDF-g-(PSSS-co-PAA) cation exchange material}

The thermodynamic properties of PVDF/PVDF- $g$ (PSSS-co-PAA) materials synthesized with different dispersion systems were analyzed by STA, as shown in Figure 2. Figure 2a and Figure $2 b$ show the STA curves of $\mathrm{P} 1$ and $\mathrm{P} 2$, respectively. The thermogravimetric (TG) curves of $\mathrm{P} 1$ and $\mathrm{P} 2$ show that the materials lost weight before the temperature reached $100^{\circ} \mathrm{C}$. This was due to the loss of bound water in the modified material, which confirmed that the modified materials were hydrophilic. Both P1 and P2 had relatively unambiguous melting points within the range of $150 \sim 160^{\circ} \mathrm{C}$ and had thermal decomposition temperatures of approximately $400 \sim 420^{\circ} \mathrm{C}$. Both P1 and $\mathrm{P} 2$ exhibited only a single melting peak at temperatures above $100^{\circ} \mathrm{C}$. This proved that the modified material had good compatibility with PVDF. P1 had a significant weight loss of approximately 5 8\% before $100^{\circ} \mathrm{C}$ and lost weight steadily as the temperature was increased beyond $100^{\circ} \mathrm{C}$, which indicated that the P1 material contained impurities, including water and low molecular weight polymers, with a broad molecular weight distribution. Both P1 and P2 had inflection points ranging within $440 \sim 460^{\circ} \mathrm{C}$. This was caused by the different thermal decomposition temperatures of the blends. Compared with P1, P2 reached its thermal decomposition temperature without an individual significant weight loss, showing an overall smooth curve. The results showed that P2, with a high molecular weight, reacted in the DMF dispersion system more completely than P1.

\subsection{Characterization of the PVDF/PVDF-g-(PSSS-co-PAA) cation exchange material}

$\mathrm{P} 1$ and $\mathrm{P} 2$ were dissolved in DMF to prepare the casting solutions. The casting solution was placed in a watch glass and dried at $60^{\circ} \mathrm{C}$ to obtain solid
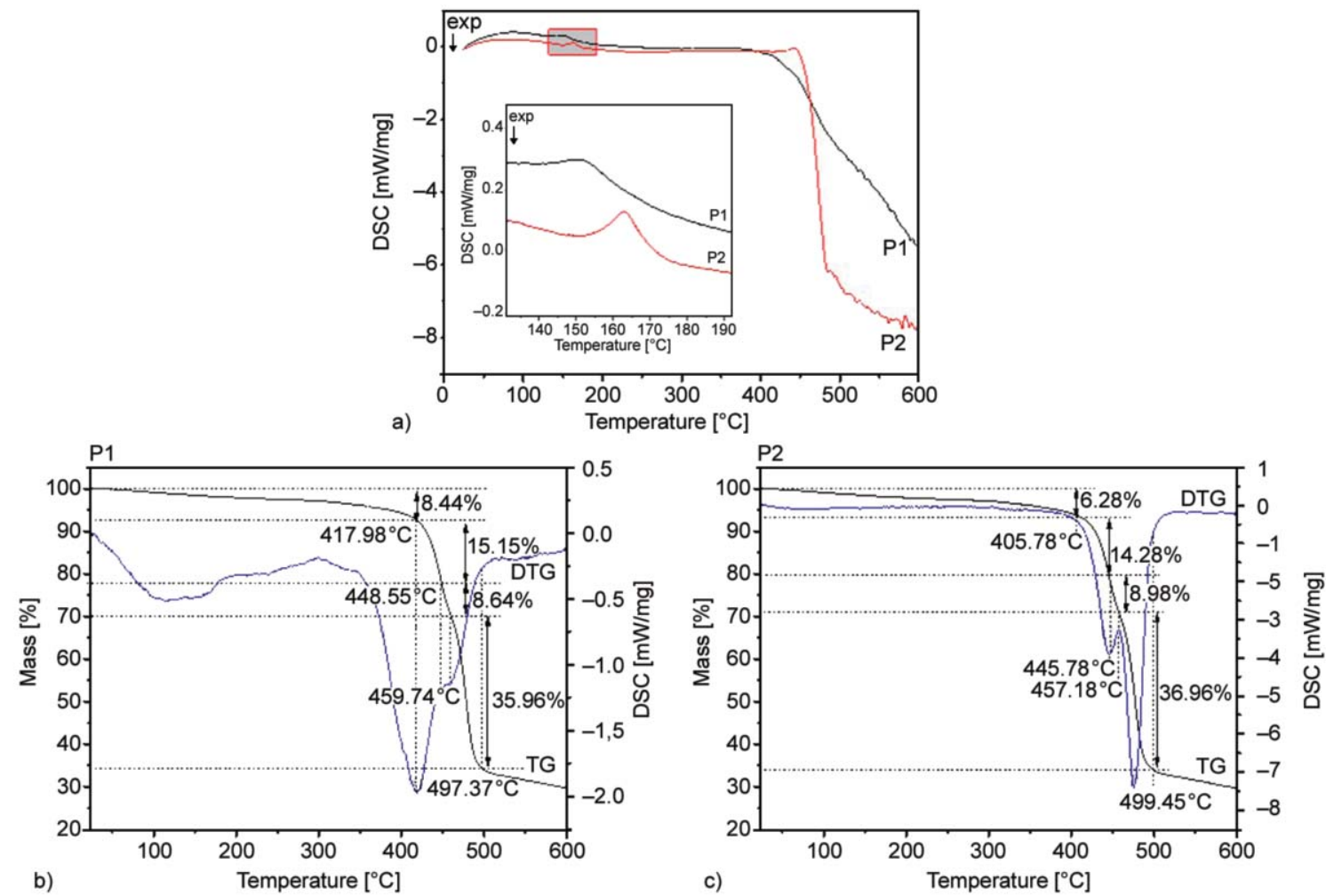

Figure 2. DSC (a) and STA of P1 (b) and P2 (c). 
blocks, which were washed repeatedly with dilute hydrochloric acid solution and then washed with deionized water to a conductivity of $100 \mathrm{mS} / \mathrm{m}$ or less. The water absorption and water absorption expansion rates were determined after $24 \mathrm{~h}$ of immersion in deionized water. The formulas for the exchange capacity, water content and volume expansion rate are as Equations (1)-(4):

Ion exchange capacity $=\frac{c_{0} \cdot V}{m(1-w)}$

where $c_{0}$ is the concentration of $\mathrm{NaOH}$ solution $0.1 \mathrm{~mol} / \mathrm{l}, V$ the volume of $\mathrm{NaOH}$ solution, $m$ is the mass of the sample, $w$ is the moisture content of the sample (see Equation (2)):

$w=\frac{m_{\mathrm{wet}}-m_{\mathrm{dry}}}{m_{\mathrm{wet}}}$

Water content $=\frac{m_{\mathrm{wet}}-m_{\mathrm{dry}}}{m_{\mathrm{dry}}}, m-$ mass

Water absorption expansion rate $=\frac{d_{\mathrm{wet}}-d_{\mathrm{dry}}}{d_{\mathrm{dry}}}$,

$$
d \text {-thickness }
$$

Table 1 shows that the water absorption of the material was controlled at approximately $10 \%$. The water swelling was limited to $13 \%$, which enhanced the structural stability of the material in the aqueous phase. The PAA used in membrane preparation swells in water, which greatly damages the dimensional stability of the membrane. The PVDF was modified by graft copolymerization, and the covalent bonds formed with hydrophilic materials enhanced the hydrophilicity and compatibility of the modified material and PVDF. Grafting effectively maintained the hydrophilicity of the modified material and limited the expansion of the material, reducing its water absorption.

\subsection{Morphology of the}

\section{PVDF/PVDF-g-(PSSS-co-PAA) cation} exchange material and membrane

The morphology of the PVDF/PVDF-g-(PSSS-coPAA) material and the effects of different synthesis processes on the properties of the modified material were analyzed by SEM, as shown in Figure 3. P1 had a uniform particle size distribution between 200 300 nm. In P2, prepared from a uniform dispersion in DMF, the molecular chains of the modified solid material obtained by phase conversion were tightly entangled. The membrane-forming properties of P2 were better than those of P1. As shown in Figure $3 \mathrm{c}$ and Figure $3 \mathrm{~d}$, the particles of M1 retained their spherical conformation with an average diameter of approximately 200 300 $\mathrm{nm}$ and remained relatively intact after membrane formation. During dissolution, P1 showed strong solvent resistance, and after $4 \mathrm{~h}$, this material had dissolved to approximately only $5 \mathrm{wt} \%$ in DMF, which is less concentrated than the casting solution of M2 (10 wt $\%)$ prepared in DMF. Thus, the solubility data and surface SEM images confirmed that there was a layer of hydrophilic PSSS-co-PAA coating the particles. The hydrophilic layer effectively prevented the dissolution of the modified material. Compared with the molecular entanglement of M1, that of M2 was more apparent. The membrane-forming process was considered in the context of the theory of phase equilibrium and the crystallization principles of polymer solutions. Above all, unreacted alkali-treated PVDF precipitated as the polymerization proceeded. Then, alkalitreated PVDF and PVDF-g-(PSSS-co-PAA) were separated from the reaction mixture, and further polymerization was halted. Besides, a polymer model could be developed in which the organic material was coated with a hydrophilic layer. Based on this model, membrane formation could be attributed to two factors: entanglement of the macromolecular chains and intermolecular hydrogen bonds. The difference in the molecular entanglements between M1 and M2 could be attributed to their solid contents. The molecular chains of M1 were coated in a hydrophilic layer before entanglement.

\section{Conclusions}

A PVDF/PVDF-g-(PSSS-co-PAA) graft copolymerization-blending cation exchange material was synthesized from different dispersion systems, either deionized water or DMF. The material had a stable exchange capacity of $1.7 \mathrm{mmol} / \mathrm{g}$, and this parameter

Table 1. Parameters obtained from $\mathrm{P} 1$ and $\mathrm{P} 2$.

\begin{tabular}{|l|c|c|c|c|}
\hline & $\begin{array}{c}\text { Ion exchange capacity } \\
{[\mathbf{m m o l} / \mathbf{g}]}\end{array}$ & $\begin{array}{c}\text { Density } \\
{\left[\mathbf{g} / \mathbf{c m}^{-\mathbf{1}}\right]}\end{array}$ & $\begin{array}{c}\text { Water content } \\
{[\%]}\end{array}$ & $\begin{array}{c}\text { Volume expansion rate } \\
{[\%]}\end{array}$ \\
\hline P1 & 0.67 & 0.33 & 5.7 & 6.24 \\
\hline P2 & 1.73 & 1.31 & 11.21 & 12.87 \\
\hline
\end{tabular}




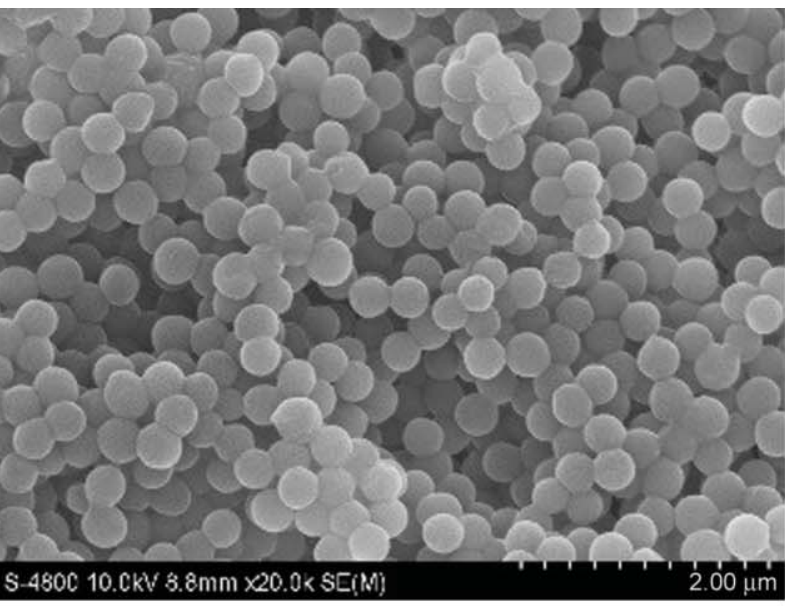

a)

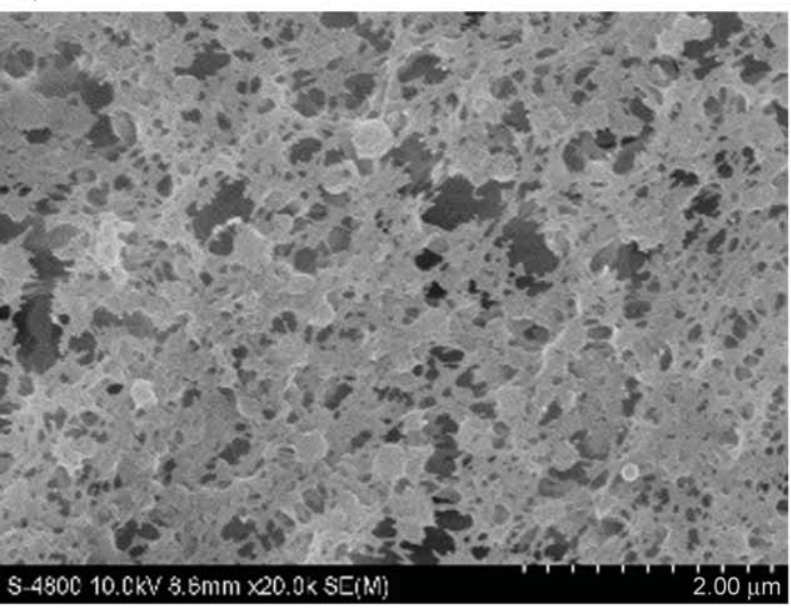

c)

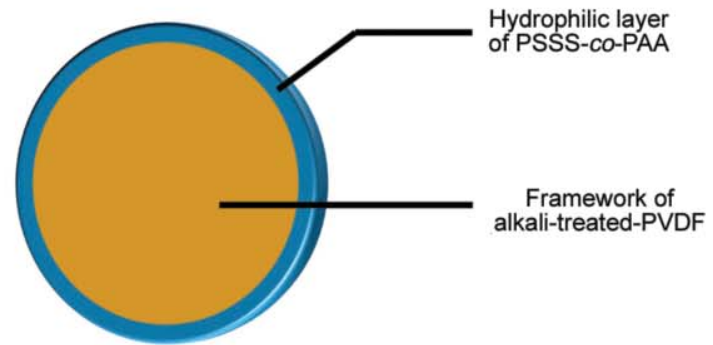

e)

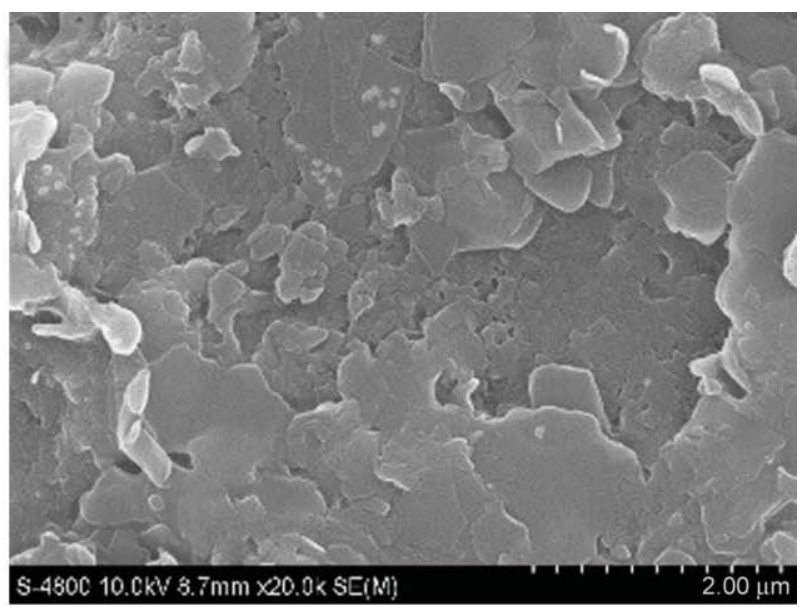

b)

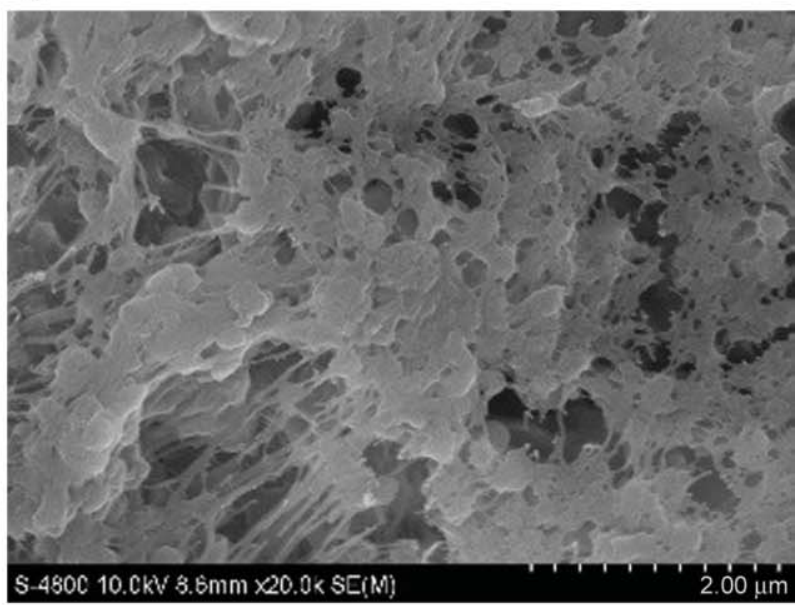

d)

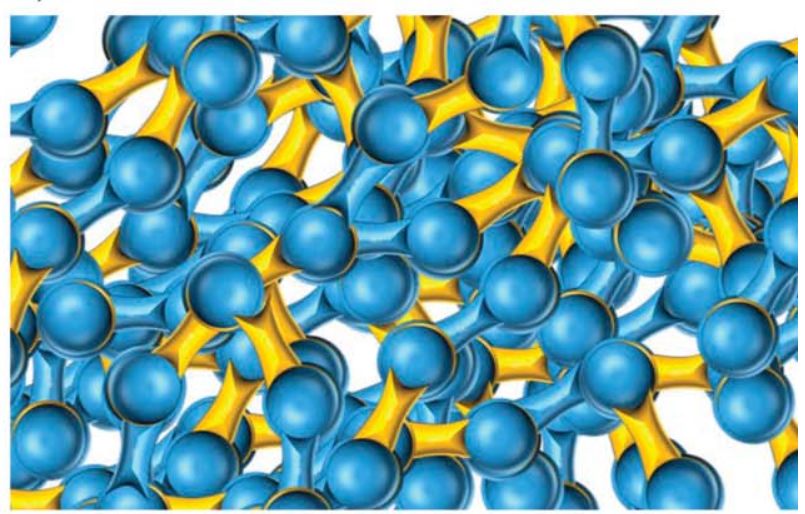

f)

Figure 3. Surface SEM images of (a) P1 synthesized in deionized water, (b) P2 synthesized in DMF, (c) M1 and (d) M2. The profile map of the P1 macromolecule model is presented in panel (e), and the macromolecule model of the PVDF/PVDF- $g$-(PSSS-co-PAA) CEM is presented in panel (f).

could be flexibly tuned. The water absorption of the material was effectively controlled within $12 \%$. Water swelling was maintained at approximately $11 \%$. The material synthesized from the deionized water dispersion system showed good structural regularity with a uniform particle size distribution within 200 300 $\mathrm{nm}$. The results provide a good reference for the independent design of hydrophilic channels, water channels, and ion channels in ion-exchange membranes. The material synthesized from DMF had good thermostability. The material was macroscopically homogeneous, and it showed good compatibility with PVDF without phase separation as a result of the thermodynamic properties. 


\section{Acknowledgements}

This work was supported by the Key Laboratory of Marine Chemistry Theory and Technology (MCTL), Ministry of Education, Ocean University of China. This work was supported by the Key Research Project of Shandong Province (No. 2018CXGC1003), and the Young Taishan Scholars Program of Shandong Province.

\section{References}

[1] Lebedeva O. V., Pozhidaev Y. N., Malakhova E. A., Raskulova T. V., Chesnokova A. N., Kulshrestha V., Pozdnyakov A. S.: Sodium $p$-styrene sulfonate-1vinylimidazole copolymers for acid-base proton-exchange membranes. Membranes and Membrane Technologies, 2, 76-84 (2020).

https://doi.org/10.1134/s2517751620020079

[2] Litmanovich E. A., Kotova E. V., Efremov V. V.: Dilutesemidilute regime crossover in aqueous solutions of poly(acrylic acid)-sodium poly(styrene sulfonate) mixtures. Colloid and Polymer Science, 297, 371-385 (2019). https://doi.org/10.1007/s00396-018-4429-1

[3] Sharma P. P., Gupta A. K., Kulshrestha V., Sharma S.: Sodium styrene sulfonate-co-methyl methacrylate-based proton conducting membranes for electrochemical energy applications. Separation Science and Technology, 54, 370-376 (2019). https://doi.org/10.1080/01496395.2018.1490324

[4] Baskoro F., Kumar S. R., Lue S. J.: Grafting thin layered graphene oxide onto the surface of nonwoven/ PVDF-PAA composite membrane for efficient dye and macromolecule separations. Nanomaterials, 10, 792/1792/15 (2020).

https://doi.org/10.3390/nano10040792

[5] Laruelle G., Nicol E., Ameduri B., Tassin J-F., Ajellal N.: Synthesis of poly(vinylidene fluoride)- $b$-poly(styrene sulfonate) block copolymers by controlled radical polymerizations. Journal of Polymer Science Part A: Polymer Chemistry, 49, 3960-3969 (2011).

https://doi.org/10.1002/pola.24836

[6] Sermili S., Eisen M. S.: Using atom transfer radical polymerization for the synthesis of grafted PVDF copolymers towards the synthesis of membranes. Israel Journal of Chemistry, 52, 347-358 (2012).

https://doi.org/10.1002/ijch.201100126
[7] Seo D-W., Lim Y-D., Lee S-H., Kang K-M., Lee K-S., Kim W-G.: Preparation and properties of hydroxide conducting membrane of poly(tetra phenyl ether sulfone) containing pendant quaternary ammonium hydroxide group for alkaline fuel cell application. in '2012 $12^{\text {th }}$ IEEE International Conference on Nanotechnology. Birmingham, UK' 1-6 (2012).

https://doi.org/10.1109/nano.2012.6321944

[8] Brewis D. M., Mathieson I., Sutherland I., Cayless R. A., Dahm R. H.: Pretreatment of poly(vinyl fluoride) and poly(vinylidene fluoride) with potassium hydroxide. International Journal of Adhesion and Adhesives, 16, 87-95 (1996). https://doi.org/10.1016/0143-7496(95)00053-4

[9] Cai T., Neoh K. G., Kang E. T., Teo S. L. M.: Surfacefunctionalized and surface-functionalizable poly(vinylidene fluoride) graft copolymer membranes via click chemistry and atom transfer radical polymerization. Langmuir, 27, 2936-2945 (2011).

https://doi.org/10.1021/la2001514

[10] Hester J. F., Banerjee P., Won Y-Y., Akthakul A., Acar M. H., Mayes A. M.: ATRP of amphiphilic graft copolymers based on PVDF and their use as membrane additives. Macromolecules, 35, 7652-7661 (2002). https://doi.org/10.1021/ma0122270

[11] Li Y., Wang H., Chu L., Zhu J., Xia S., Chen W., Wang G., Wang S.: pH-responsive gating characteristics of PAAC-grafted porous polyvinylidene fluoride membranes (in Chinese). Journal of Chemical Industry and Engineering, 55, 1016-1020 (2004).

[12] Alexowsky C., Bojarska M., Ulbricht M.: Porous poly (vinylidene fluoride) membranes with tailored properties by fast and scalable non-solvent vapor induced phase separation. Journal of Membrane Science, 577, 69-78 (2019).

https://doi.org/10.1016/j.memsci.2019.01.033

[13] Safarpour M., Khataee A., Vatanpour V.: Preparation of a novel polyvinylidene fluoride (PVDF) ultrafiltration membrane modified with reduced graphene oxide/titanium dioxide $\left(\mathrm{TiO}_{2}\right)$ nanocomposite with enhanced hydrophilicity and antifouling properties. Industrial and Engineering Chemistry Research, 53, 13370-13382 (2014).

https://doi.org/10.1021/ie502407g 